\title{
Non-invasive imaging techniques for the in vivo diagnosis of Bowen's disease: Three case reports
}

\author{
SIMONA LAURA IANOȘI ${ }^{1 *}$, ALEXANDRA BATANI $^{2^{*}}$, MIHAELA ADRIANA ILIE $^{3,4^{*}}$, MIRCEA TAMPA $^{5^{*}}$, \\ SIMONA-ROXANA GEORGESCU ${ }^{5^{*}}$, SABINA ZURAC ${ }^{6,7^{*}}$, DANIEL BODA $^{3,8^{*}}$, NICOLAE GABRIEL IANOSI $^{9^{*}}$, \\ DANIELA NEAGOE ${ }^{10^{*}}$, DANIELA CALINA $^{11^{*}}$, CRISTINA TUTUNARU $^{1 *}$ and CARUNTU CONSTANTIN ${ }^{8,12^{*}}$ \\ ${ }^{1}$ Department of Dermatology, University of Medicine and Pharmacy of Craiova, Emergency County Hospital, \\ 200349 Craiova; ${ }^{2}$ Department of Dermatology, Colentina Clinical Hospital, 020125 Bucharest; \\ ${ }^{3}$ Dermatology Research Laboratory and Departments of ${ }^{4}$ Biochemistry, ${ }^{5}$ Dermatology and ${ }^{6}$ Pathology, \\ 'Carol Davila' University of Medicine and Pharmacy, 050474 Bucharest; ${ }^{7}$ Department of Pathology, \\ Colentina Clinical Hospital, 020125 Bucharest; ${ }^{8}$ Department of Dermatology, 'Prof. N. Paulescu' National Institute \\ of Diabetes, Nutrition and Metabolic Diseases, 011233 Bucharest; Departments of ${ }^{9}$ Surgery, ${ }^{10}$ Internal Medicine \\ and ${ }^{11}$ Pharmacology, University of Medicine and Pharmacy, 200349 Craiova; ${ }^{12}$ Department of Physiology, \\ 'Carol Davila' University of Medicine and Pharmacy, 050474 Bucharest, Romania
}

Received September 11, 2018; Accepted December 18, 2018

DOI: $10.3892 / 01.2019 .10079$

\begin{abstract}
Bowen's disease (BD) is a relatively frequent nonmelanoma skin cancer occurring mostly in elderly people. Until now, the usual way to establish the diagnosis is histopathological examination of a skin biopsy. Dermoscopy and reflectance confocal microscopy (RCM) are modern alternative methods that can be used as quick and non-invasive diagnostic techniques and as follow-up instruments in cases in which a conservative treatment is chosen for the management of BD. There are no very specific dermoscopic criteria for the diagnosis of this disease, but some dermoscopic features (scaly surface, vascular structures and pigmentation) can be found more frequent and can be helpful for the diagnosis. RCM of BD shows an acanthotic epidermis with two types of targetoid cells: the first, a large cell with bright center and dark peripheral halo, the second, a cell with dark center and a bright rim surrounded by a dark hallo, related with dyskeratotic cells on histological examination. BD management could be improved by using non-invasive, in vivo imaging techniques that allow a fast and easy diagnosis and can be used as follow-up tools. However, larger studies are necessary for the validation of our observations.
\end{abstract}

Correspondence to: Dr Daniel Boda, Dermatology Research Laboratory, 'Carol Davila' University of Medicine and Pharmacy, 8 Eroii Sanitari Boulevard, 050474 Bucharest, Romania

E-mail: daniel.boda@yahoo.com

*Contributed equally

Key words: Bowen's disease, dermoscopy, reflectance confocal microscopy, in vivo, non-invasive, diagnosis

\section{Introduction}

Bowen's disease (BD), often considered an in situ squamous cell carcinoma (SCC) is a relatively frequent nonmelanoma skin cancer occurring mostly in elderly people with photodamaged skin (1). In 3-5\% of cases it may progress into invasive SCC, and $10 \%$ of invasive tumors may metastasize $(2,3)$. Clinically, it presents most commonly as a slowly growing erythematous or light brown papule or plaque, flat or slightly elevated, with a scaly surface. Due to the lack of distinctive features it can often pose as a diagnostic dilemma. BD has also a pigmented variant that may share overlapping clinical features with melanoma $(4,5)$.

Dermoscopy was shown to be useful in the assessment of pigmented and nonpigmented skin tumors as it allows a rapid and non-invasive recognition of vascular or pigmented structures that are not visible to the naked eye $(6,7)$.

Another imaging technique that allows a non-invasive and in real-time examination of pigmented and nonpigmented skin lesions is the reflectance confocal microscopy (RCM). Its usefulness in the assessment of tumoral (8-10), inflammatory (11-14) or infectious skin lesions has been proven by extensive research $(15,16)$.

In this study, we highlight the usefulness of combining the two imaging methods for a non-invasive and rapid diagnosis of BD. Therefore, we present 3 cases of BD that we have examined dermoscopically and by RCM technique and correlated to the corresponding histological examination.

\section{Materials and methods}

The study was conducted in the Department of Dermatology, 'Prof. N.C. Paulescu' National Institute of Diabetes, Nutrition and Metabolic Diseases (Bucharest, Romania) and approved by the Ethics Committee of 'Prof. N.C. Paulescu' National 

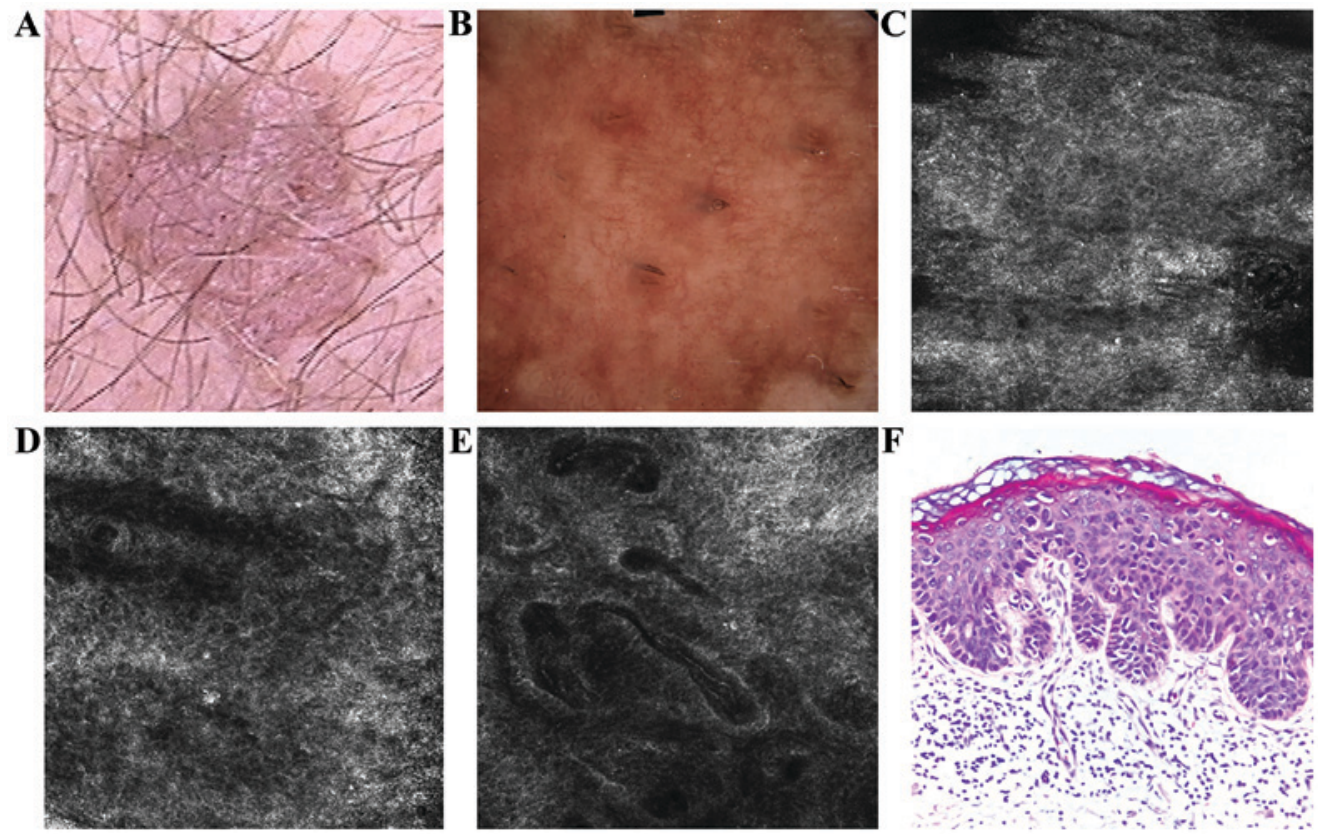

Figure 1. (A) Clinical image illustrated a well-demarcated, erythematous, scaly plaque. (B) Dermoscopic aspect of the lesion revealed vascular pattern with dotted, linear, comma-like and glomerular vessels. (C) RCM examination (500x500 $\mu \mathrm{m}$ ) of the stratum corneum showed the presence of bright, polygonal shaped structures corresponding to parakeratotic cells (D) RCM image (500x500 $\mu \mathrm{m}$ ) with chaotic appearance of the spinous layer resulted in an atypical honeycomb pattern and sparse target-like cells. (E) RCM image (500x500 $\mu \mathrm{m}$ ) of the dermal-epidermal junction revealed enlarged, irregular shaped papillae filled with dilated capillaries, with reduction of the papillary rings normally seen in healthy skin. (F) Histopathological image showed areas of parakeratosis, numerous dyskeratotic keratinocytes, moderate cellular and nuclear pleomorphism and numerous mitosis; moderate hyperemia and lymphocytic inflammatory infiltrate (hematoxylin and eosin staining; x200 magnification).

Institute of Diabetes, Nutrition and Metabolic Diseases, and informed consent was obtained from the patients. Clinical evaluation of the lesions followed the guidelines for diagnosis of BD. In each case, clinical photographs of the lesions and dermoscopic images were acquired using a digital videodermoscopy system (FotoFinder, Bad Birnbach, Germany) and the VivaScope 1500 VivaCam macro camera (Lucid Inc., Rochester, NY, USA). A commercially available in vivo confocal microscope (Vivascope 1500; Lucid Inc.) was used for RCM imaging. All lesions evaluated in our study underwent excision under local anesthesia and histopathological examination was performed after standard hematoxylin and eosin staining of the excised tissue.

\section{Case reports}

Case 1. A 78-year-old male, phototype II, presented in above-mentioned Department for the evaluation of a welldemarcated, slowly enlarging, erythematous, scaly plaque of $1.8 \times 1.6 \mathrm{~cm}$, located on the abdomen (Fig. 1A). On dermoscopic examination pink structureless areas and a polymorphous vascular pattern consisting of dotted, linear, comma-like and glomerular vessels were detected (Fig. 1B).

On RCM images, the stacking examination showed a thickened stratum corneum; the presence of polygonal, refractile structures in this layer corresponds to nucleated corneocytes (parakeratosis) and grouped, elongated, bright structures correspond to inflammatory infiltrates (Fig. 1C). Descending to the spinous layer, an atypical honeycomb pattern due to great variations in cell and nuclear morphology, and rare targetoid cells were identified (Fig. 1D). At the dermo-epidermal junction, there was an increased number of dermal papillae of different sizes and shapes, some of them connecting; the bright hallo surrounding dermal papillae seen in healthy skin, corresponding to basal cells, was diminished in the lesion examined. Dilated and tortuous capillaries were seen as large, dark, round to oval or canallicular structures containing bright blood cells (Fig. 1E)

The biopsy showed areas of parakeratosis with absence of underlying granular layer, alternating with areas of orthokeratosis; the epidermis was disorganized with atypical keratinocytes with hypertrophic nuclei; moderate lymphocytic inflammatory infiltrate with rare melanophages in the superficial dermis. The histologic features were consistent with BD (Fig. 1F).

Case 2. A 76-year-old male, phototype III, presented to the above-mentioned Dermatology Department for evaluation of two slowly growing plaques situated on the lateral and dorsal aspects of the thorax respectively. The first lesion was a well-demarcated, flat plaque of $1.5 \times 1.3 \mathrm{~cm}$ with irregular borders, showing pink and brown diffuse areas and slightly scaly surface (Fig. 2A). This lesion revealed a dermoscopic pattern characterized by a diffuse, structureless light-brown pigmentation, a few dark-brown irregular distributed globules, conglomerates of glomerular vessels and some linear and dotted vessels (Fig. 2B). RCM images showed a thick stratum corneum with round to polygonal nucleated bright cells corresponding to parakeratotic korneocites and intensely refractile round structures corresponding to infiltrating neutrophils (Fig. 2C). Associated with the atypical honeycomb pattern in the spinous layer, there was a high-density 

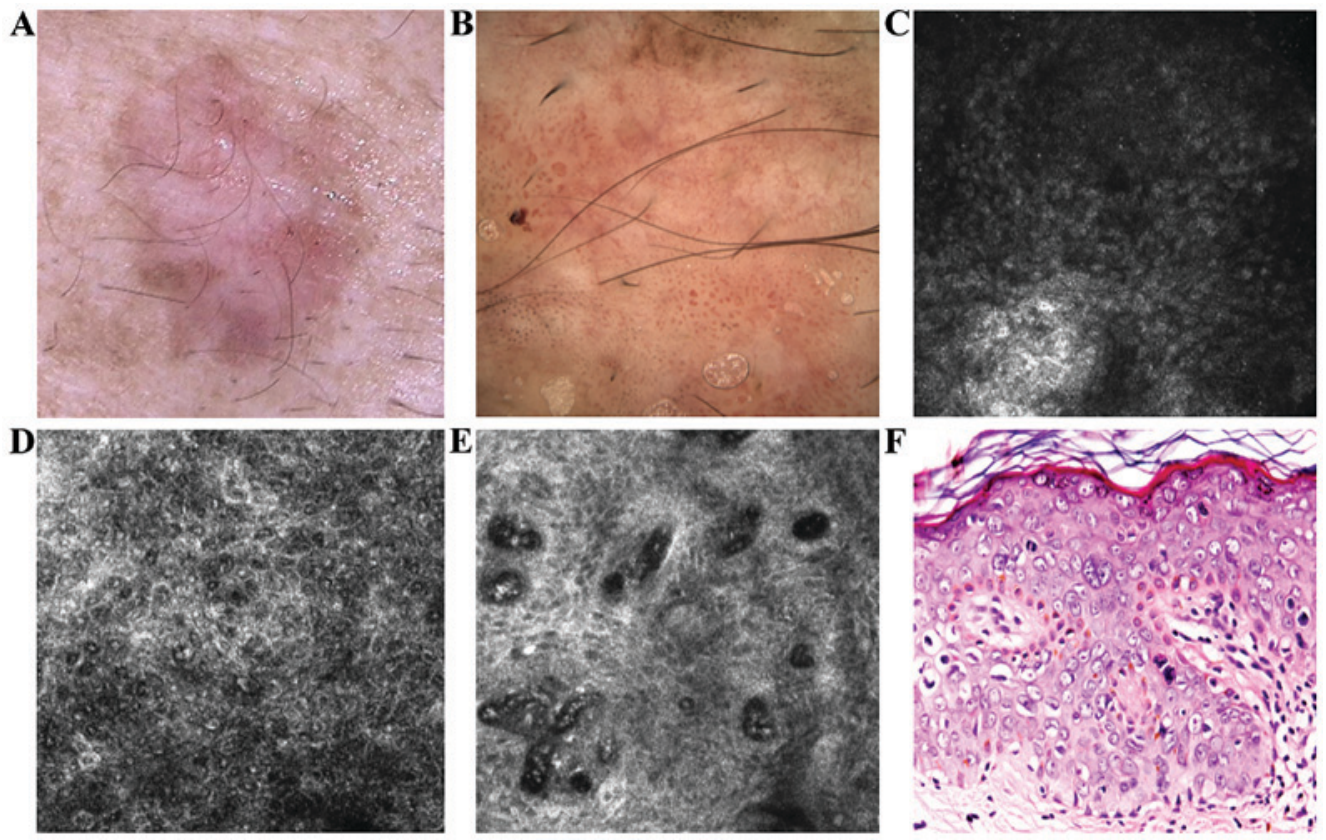

Figure 2. (A) Clinical image of a flat plaque showed pink and brown diffuse areas. (B) Corresponding dermoscopic image showed diffuse, structureless brown pigmentation, some brown globules and an atypical vascular pattern consist of glomerular, dotted and linear vessels. (C) RCM image (500x500 $\mu \mathrm{m})$ at the level of the stratum corneum where parakeratotic cells were identified as round to polygonal, refractile structures. (D) RCM image (500x500 $\mu \mathrm{m})$ revealed the atypical honeycomb pattern in the spinous layer, with various shapes and sizes of the cells and nuclei. Many of these cells have a target-like aspect, with a bright center and a dark peripheral halo. Another type of cell with targetoid aspect, with a dark center and a bright peripheral rim, was also identified in smaller numbers. (E) RCM image (500x500 $\mu \mathrm{m})$ at the level of the dermo-epidermal junction showed enlarged dermal papillae, with bizarre shapes, without the papillary rings of basal cells, inside of which are identified dilated capillaries, some captured in horizontal section, parallel to the skin. (F) Histopathological image displaying important cytonuclear pleomorphism with hypertrophy and nuclear monstrosities and atypical mitosis at different levels in epidermal thickness (hematoxylin and eosin staining; x200 magnification).
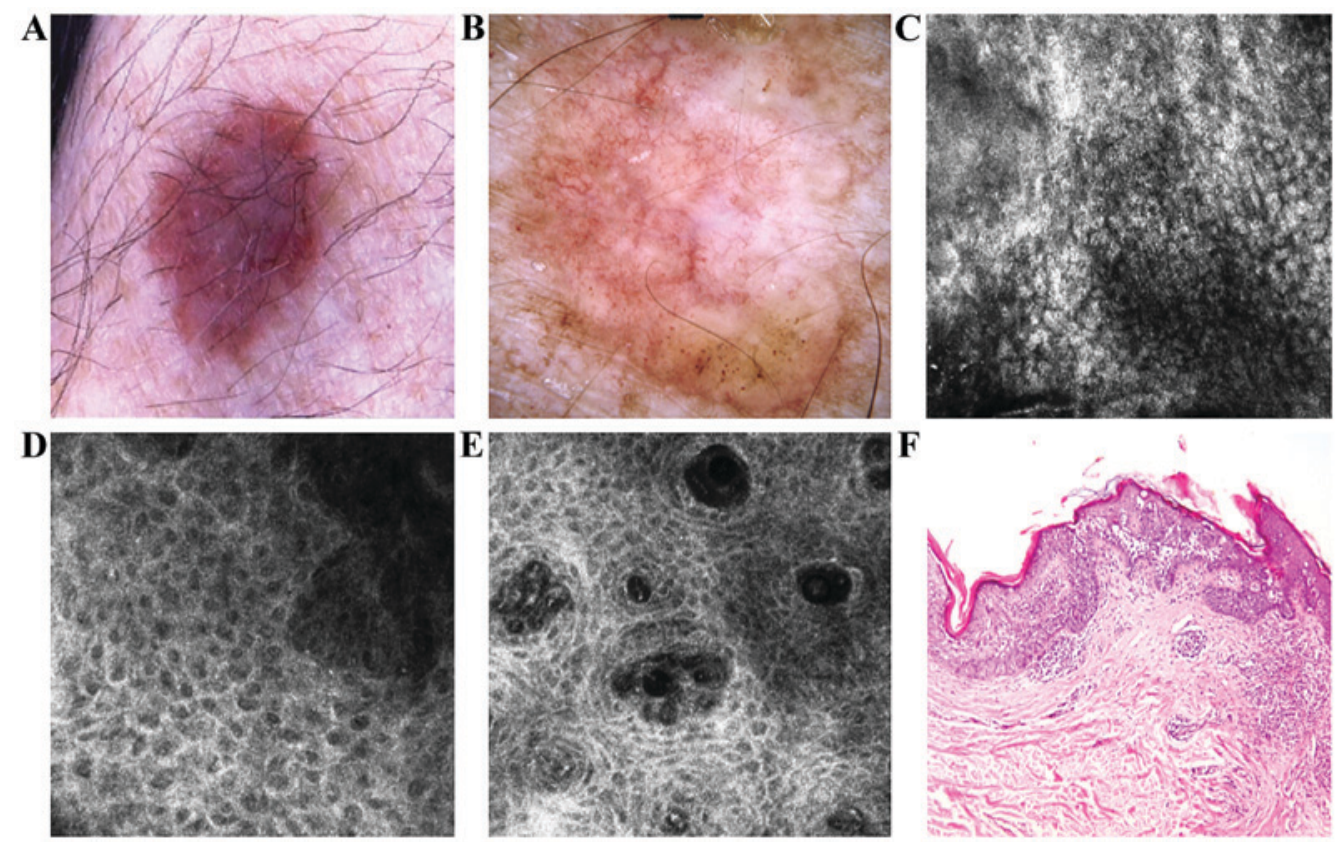

Figure 3. (A) Clinical image of a slightly variegated brown plaque with very fine scaling. (B) Dermoscopic view showed structureless asymmetric brown pigmentation with erythematous background, few brown globules and irregular network in the periphery and an atypical vascular pattern. (C) RCM examination $(500 \times 500 \mu \mathrm{m})$ revealed parakeratosis in the horny layer. (D) RCM image (500x500 $\mu \mathrm{m})$ at the level of the spinous-granular layers showed a disarrayed pattern and target-like cells. (E) RCM image $(500 \times 500 \mu \mathrm{m})$ at the dermo-epidermal junction the dermal papillae are filled with many dilated capillaries. (F) Corresponding histology with epidermal thickening due to proliferation of atypical squamous cells with cyto-nuclear atypia extended through the whole thickness of the epidermis (hematoxylin and eosin staining; x100 magnification).

of targetoid cells, recognizable as enlarged round cells with a bright center and a dark peripheral hallo or as enlarged cells with dark center surrounded by a bright rim (Fig. 2D). RCM examination of the superficial dermis exhibited dysmorphic, 

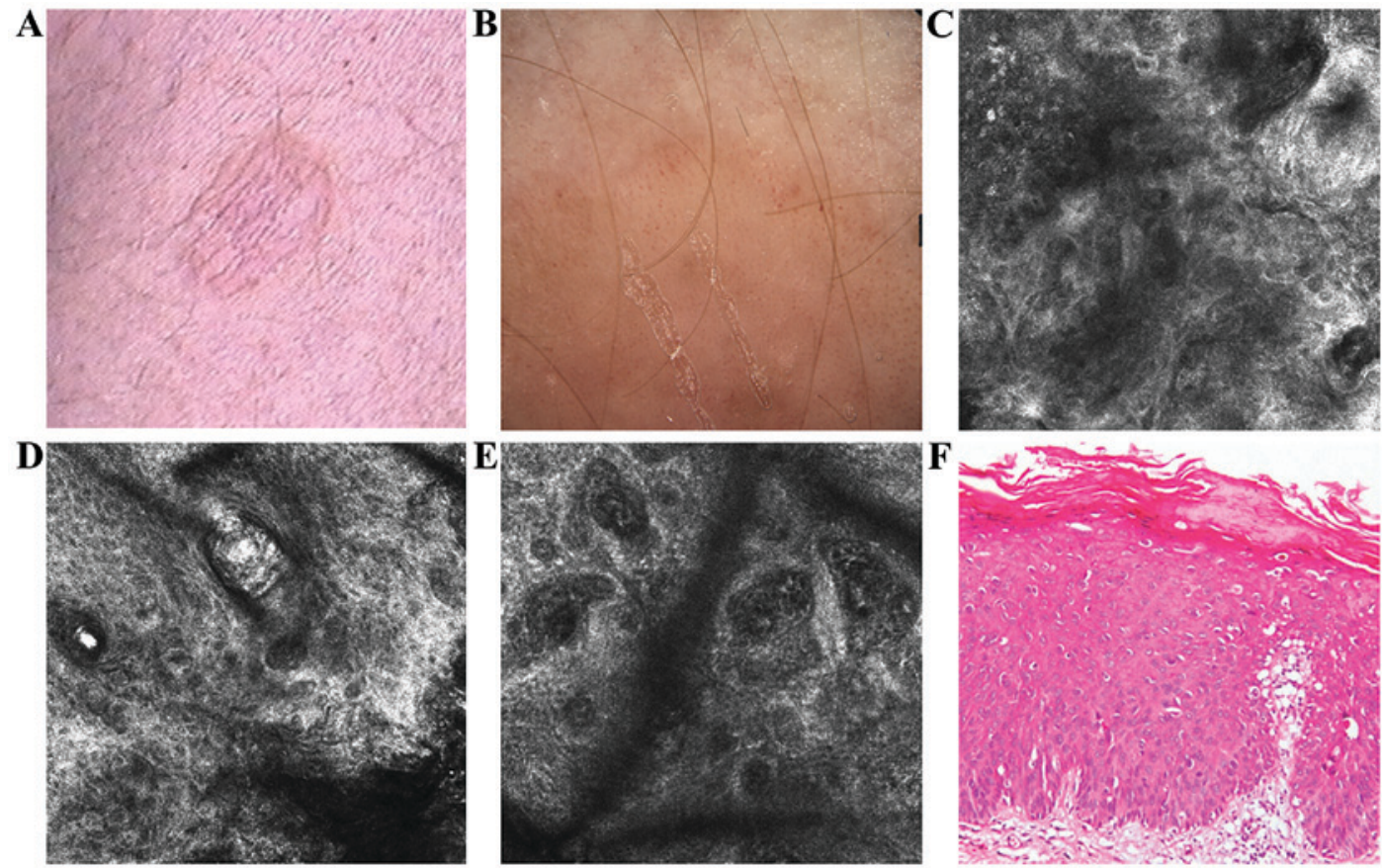

Figure 4. (A) Clinical image showed a scaly, pink and light brown, flat plaque, with well-defined borders. (B) Dermoscopic image showed coil-shaped and dotted vessels and light brown structureless areas on a pink background. (C) RCM image (500x500 $\mu \mathrm{m})$ revealed a parakeratosis area in the horny layer (upper left). (D) RCM image (500x500 $\mu \mathrm{m}$ ) at the level of the spinous layer showing an atypical honeycomb pattern. (E) RCM image (500x500 $\mu \mathrm{m})$ at the dermo-epidermal junction revealing enlarged dermal papillae with dilated and sinuous blood vessels. (F) Histopathological image showing epidermal thickening with areas of parakeratosis, atypical keratinocytes, moderate pleomorphism and moderate lymphocytic inflammatory infiltrate (hematoxylin and eosin staining; x200 magnification).

large papillae, containing dilated capillaries with sinuous trajectories parallel on the horizontal sections of the skin and with increased blood flow in real-time imaging (Fig. 2E). Histopathological examination of both lesions confirmed the diagnosis of BD (Figs. 2F and 3F).

Naked-eye examination of the second lesion revealed an irregular-shaped, well-demarcated, brown and grey plaque of $1.3 \times 0.8 \mathrm{~cm}$, with fine scales on the surface (Fig. 3A). Dermoscopic examination of the lesion revealed dark-pink and brown diffuse areas overlapped by vessels with varied sizes and shapes, mostly glomerular, dotted and commalike (Fig. 3B). RCM examination revealed a thick cornified layer with remaining parakeratotic korneocytes and extensive black areas containing multiple refractile oval and elongated cells (Fig. 3C). The atypical honeycomb pattern with high-density of targetoid cells was also identified (Fig. 3D). Descending to the dermo-epidermal junction it can be noted that the atypical honeycomb pattern is maintained in the lower layers. Enlarged papillae containing a high number of dilated vessels with intense blood flow were also revealed (Fig. 3E).

Case 3. A 56-year-old male, phototype III, without any significant personal and familial medical history, presented to the above Dermatology Department for investigation of a $2 \times 1.5 \mathrm{~cm}$ flat plaque, with well-defined borders and a slightly scaly surface, colored in shades of pink and light brown, located on the inner aspect of the left calf (Fig. 4A). The dermoscopic assessment of this skin lesion showed coil-shaped and dotted vessels on a pink to light-brown background (Fig. 4B).

$\mathrm{RCM}$ examination showed a thick horny layer with areas of parakeratosis, atypical honeycomb pattern of spinous layer (Fig. 4C and D). At the dermo-epidermal junction enlarged papillae with dilated and tortuous blood vessels were revealed (Fig. 4E). Histological examination showed typical features of BD (Fig. 4F).

Our results confirm the RCM features of $\mathrm{BD}$, showing a good correlation with dermoscopic and histopathologic findings (Table I).

\section{Discussion}

In many cases, $\mathrm{BD}$ presents as a diagnostic dilemma due to lack of specific clinical features. At present, the only way to establish diagnostic certainty of BD is by histopathological examination of a skin biopsy. Current trends in skin imaging are focused on non-invasive techniques characterized by rapidity, possibility to evaluate the evolution of the lesions over time by preserving skin integrity, lack of invasiveness and patient comfort (17). The unpredictable evolution of $\mathrm{BD}$ to invasive SCC is an important issue especially when the lesion is incorrectly managed as an erythemato-squamous inflammatory skin disease. Given that, until now, very little information related to non-invasive diagnostic techniques for $\mathrm{BD}$ can be found in literature.

In 2012, Ulrich et al examined ten cases of BD using dermoscopy and RCM achieving results that match our observations (18). In all cases they identified parakeratosis, neutrophilic inflammatory infiltrate within the stratum corneum, atypical honeycomb pattern in the spinous layer extending to the whole epidermis thickness, two types of targetoid cells and dilated, tortuous capillaries. These observations had a good correlation with histopathological examination. 
Table I. RCM features of BD and their dermoscopic and histopathologic correlations.

\begin{tabular}{|c|c|c|c|c|c|c|}
\hline \multirow[b]{2}{*}{ RCM features } & \multirow[b]{2}{*}{ Case 1} & \multicolumn{2}{|c|}{ Case 2} & \multirow[b]{2}{*}{ Case 3} & \multirow{2}{*}{$\begin{array}{l}\text { Dermoscopic } \\
\text { correlation }\end{array}$} & \multirow{2}{*}{$\begin{array}{l}\text { Histopathological } \\
\text { correlation }\end{array}$} \\
\hline & & Lesion 1 & Lesion 2 & & & \\
\hline $\begin{array}{l}\text { Polygonal, refractile structures } \\
\text { in the superior layers }\end{array}$ & + & + & + & + & - & $\begin{array}{l}\text { Parakeratotic } \\
\text { corneocites }\end{array}$ \\
\hline Atypical honeycomb pattern & + & + & + & + & - & $\begin{array}{l}\text { Cyto-nuclear } \\
\text { pleomorphism }\end{array}$ \\
\hline Targetoid cells & + & + & + & - & - & Dyskeratotic cells \\
\hline Dysmorphic, large papillae & + & + & + & + & - & Papillomatosis \\
\hline Dilated, sinuous capillaries & + & + & + & + & $\begin{array}{l}\text { Atypical vascular } \\
\text { pattern }\end{array}$ & $\begin{array}{l}\text { Dilated } \\
\text { capillaries }\end{array}$ \\
\hline
\end{tabular}

RCM, reflectance confocal microscopy.

Another study from 2016 highlights through a case report the usefulness of RCM technique in distinguishing between a lesion that presented clinically and dermoscopically as a pigmented BD but histopathological examination proved to be a lightly pigmented melanoma (19). The pigmented version of BD is often a diagnostic challenge whereas the differential diagnosis with melanoma is crucial (20-23).

There are no specific dermoscopic criteria described in the literature for the diagnosis of BD but some features such as scaly surface, vascular structures and pigmentation defined by small brown globules with patchy distribution, reticular pigmentation and homogeneous gray brownish pigmentation, can be found more frequently and although not specific, they can be helpful in making a diagnosis. Moreover, the absence of specific dermoscopic features of the melanocytic lesions was noted such as pigmented network, irregular lines, blue white structures and parallel pattern (21-23).

The typical or atypical vascular component is well-represented BD and consists of regular dotted vessels, linear, bushy, arborizing, hairpin-like vessels and milky red globules. The vascular structures may appear as dots, clods or lines. If they appear as lines, vessels may be straight, curved, looped (one major bend of approximately 180 degrees), serpentine (multiple bends), helical (multiple bends along a central axis), or coiled (multiple tight bends convoluted compactly). The last type is the same as the so-called 'glomerular' vessels described by other authors and correspond to dilated vessels found in the papillary dermis $(24,25)$. Although the arrangement of the dots or vessels as lines is characteristic for BD, it has been described that the linear or serpiginous arrangement of coiled vessels is highly specific for clear cell acanthoma. However, the clinical differential diagnosis of the two is easy. Other authors consider this arrangement in lines of coiled vessels and/or dots (gray and/or brown) is a specific feature of BD and allows differential diagnosis from melanoma (5). The neoangiogenesis (neovascularization) process that was quite common and prominent is clearly correlated with the neoplastic nature of BD (26).

Regarding the amount of pigmentation, BD can appear under different dermoscopic aspects, varying from the absence of pigmented network or scarcely pigmented like a pseudo-network, irregular diffuse pigmentation or pigmented areas, irregularly distributed dots and globules, or focal/multifocal hypopigmentation. The colors noted by dermoscopy may be due to melanin or other pigments, like hemoglobin (5). The most frequent aspect was a structureless pattern seen in almost half of the cases and shades of brown represented the predominant color. There were variants of this structureless brown pattern with few circles and dots on a brown structural background (5). This pattern is the least specific dermoscopically for BD and when combined with no vessels can lead to misdiagnosis of this condition by dermoscopy. Another common appearance is the asymmetric combination of the patterns, structureless and dots. Usually, the structureless areas are hypopigmented in comparison to the brown found in one-pattern lesions. Furthermore, these areas are mostly located eccentrically. When brown and gray pigmentation is intense, the lesion can be mistaken as a melanoma (27-29).

In a $40-\mathrm{BD}$ cases study performed by Bugatti et al, all the patients presented a multicomponent pattern but the most frequent dermoscopic features were the atypical vascular structures represented by dotted vessels followed by absence of pigmented network and focal/multifocal hypopigmentation (30).

In another study performed on 52 patients with pigmented $\mathrm{BD}$ in Australia, the authors used Kittler's pattern for dermoscopic analysis which is a standardized description of pigmented lesions based on patterns, colors and clues (5,26-31). The most frequent pattern was asymmetrically structureless combined or not with dots. With regard to pigmentation, brown color was the most frequent, followed by a combination of brown and gray and in a few cases only gray.

Moreover, hypopigmented (pink, skin colored, or white) structureless zones were present in $67.2 \%$ of the lesions. In all cases of pigmented $\mathrm{BD}$, the distribution of pigment resulted in a variegated appearance. An important clue was brown or gray dots in a linear arrangement seen in $21.2 \%$ of cases and most often at the periphery with the lines oriented radially.

There is a correlation between the presence or absence of melanophages in the dermis and dermatoscopic aspects. The absence of melanophages at histopathological examination was correlated with the absence of the gray dots in dermoscopy. If 
melanophages were present, the ability to identify gray dots in dermoscopy increases with their histopathological density. As regards inflammation, even if it was abundent in over $45 \%$ of cases, the authors were not able to highlight any correlation with dermoscopic features (5).

In $\mathrm{BD}$, dermoscopy can be used as a valuable follow-up tool. In one case, glomerular vessels disappeared after laser treatment and it correlates histologically to the decreased vascularity in the dermal papillae (31). The occurrence of glomerular vessels in BD seen by dermoscopy is unique among skin tumors and therefore can indicate a proper treatment of this condition $(24,26,30)$. Furthermore, there was a generalized decrease in the degree of pigmentation and the absence of small brown globules and reticular pigmentation after laser therapy that corresponded histologically to the loss of melanophages in the papillary dermis (31).

RCM is relatively new imaging tool applied for non-invasive evaluation of the skin, especially in dermato-oncology that provides horizontal scanning of the skin at nearly histological resolution. In normal skin, vascular structures and blood vessels can be visualized in real-time, high resolution examination by RCM. In the dermis, they appear as dark spaces transited by small bright blood cells, process named leukocyte rolling $(32,33)$. The vessels arranged horizontally and perpendicularly to the surface are visualized as canalicular or, respectively, dark spaces. RCM can also be used to quantify the size and the density of the vessels and the flow intensity. Additional information regarding the diagnosis and monitoring of the nonpigmented tumoral lesions can result from the vascular morphology and the pattern's distribution.

Some characteristic features for the diagnosis of nonpigmented tumoral lesions of the skin have been described (32). For in situ SCC, RCM shows the vessels traversing through the papilla perpendicular to the horizontal confocal plane, appearing round in cross section $(17,32,33)$. The vascular component is prominent due to increased number, density and vasodilatation of the capillaries that usually traverse the dermal papillae. In real-time an increased blood flow was noted.

On RCM, BD shows an acanthotic epidermis with an atypical/disarranged honey-comb pattern with grainy appearance with variation in cell and nuclear morphology. Two types of targetoid cells were identified: the first, a large cell with bright center and dark peripheral halo, the second a cell with dark center and a bright rim surrounded by a dark halo. At the dermoepidermal junction, non-edged, multiple dermal papillae of different sizes with weakly refractive dense and sparse nests and nucleated atypical cells and dilated round blood vessels were observed (18).

The histopathological examination of BD shows characteristic features: proliferation of atypical keratinocytes with marked nucleocytoplasmic pleomorphism (poikilocarynosis), frequent atypical mitoses and dyskeratosis. There is a correlation between the histological features and the ones observed on RCM. For example, the two types of targetoid cells seen by RCM relate to different morphological types of dyskeratotic cells observed histological. In addition, large cells with bright center surrounded by a dark halo correspond to dyskeratotic keratinocytes separated by a clear retraction halo seen on hematoxylin and eosin sections (33). The second type of targe- toid cells correlates histological to dyskeratotic keratinocytes with pycnotic nucleus. Cells with aggregated nuclei on RCM corresponded to multinucleated keratinocytes seen on hematoxylin and eosin sections. The atypical honeycomb pattern corresponded histologically to poikilocarynosis. Round to oval blood vessels ad tortuous S-shaped structures seen on RCM relate to round/oval vessel histologically. Small, bright cells found in the superficial dermis correspond to inflammatory cells seen on histologic sections.

The vascular structure was described in numerous studies (34). Therefore, the vascular morphology was defined as 6 types of vessels: straight linear, curved linear, tubular/ canalicular round and polymorph and branching vessels. RCM showed in the majority of cases the presence of curved linear vessels as equivalent glomerular vessels, regular and irregular hairpin vessels in dermoscopic examination. Many studies showed slightly elongated round vessels in dermal papillae in the superficial dermis on RCM examination $(35,36)$. In a study by Incel et al performed on 122 tumoral lesions, curved linear $\mathrm{S}$-shaped vessels in actinic keratosis/BD were seen in $93.3 \%$ of cases (34). All BD lesions showed this type of vessels as an equivalent of glomerular vessels on dermoscopy (37). The tumoral lesions with vascular polymorphism were associated with higher rates of malignancy (34).

In a study of 10 cases, RCM showed superficial epidermal disruption in all cases, with single, detached and nucleated keratinocytes corresponding to parakeratosis (37). In 7 out of 10 cases, neutrophils within the corneum layer were observed. Also, an atypical honey-comb pattern with variations in cell and nuclear morphology was seen in all cases at the granular/spinous epidermal layers. In this study, in half of the cases targetoid cells with bright center and dark peripheral halo were seen. In all cases, in the center of the dermal papillae, round to oval blood vessels with prominent tortuosity were noted. S-shaped blood vessels were observed in 7 cases. A retrospective analysis of the vertical sections, showed the round to oval vessels within the superficial papillary dermis and S shaped vessels at the lower papillary dermis. In 5 out of 10 cases, small, round, bright cells corresponding to inflammatory cells were observed.

In BD a disorganized pattern of the epidermis with numerous hyper reflective dendritic intraepithelial cells compatible with Langerhans cells and occasional ones around the papillae at the dermo-epidermal junction were revealed on RCM examination $(38,39)$. In time, Langerhans cells had totally disappeared after laser treatment confirming complete remission of the tumor. Hence, RCM may be regarded as a complementary technique for assessing treatment efficacy.

Besides its usefulness as a quick and non-invasive diagnostic method, RCM may be used as a follow-up instrument in cases in which a conservative treatment is chosen for the management of BD. Due to its advantage in preserving skin integrity, allowing multiple examinations of the same area over time, the response to conservative therapy regimen can be evaluated, complete healing can be concluded and relapses can be tracked, aspects which currently are not possible. However, comprehensive studies are required in order to establish criteria applicable in everyday practice.

In conclusion, in this study we demonstrated that BD management could be improved by using non-invasive, in vivo imaging techniques that allow a fast and easy diagnosis and to 
follow-up the treatment efficacy. However, larger studies are necessary for the validation of our observations.

\section{Acknowledgements}

Not applicable.

\section{Funding}

This study was partially supported by a grant of Romanian Ministry of Research and Innovation, CCCDI-UEFISCDI (project no. 61PCCDI/2018 PN-III-P1-1.2-PCCDI-2017-0341) within PNCDI-III.

\section{Availability of data and materials}

The datasets used and/or analyzed during the current study are available from the corresponding author on reasonable request.

\section{Authors' contributions}

SLI, DB, CC were responsible for the design of the study and interpretation of the data. They also have revised critically the manuscript for important intellectual content. AB, MAI, SZ were responsible for the data acquisition, selection and analysis and clinical interpretation of the data. MT, SRG, NGI, DN, DC, CT were responsible for the data analysis and interpretation. All authors contributed to the writing of the manuscript. All authors read and approved the final version of manuscript.

\section{Ethics approval and consent to participate}

The study was conducted in the Department of Dermatology, 'Prof. N.C. Paulescu' National Institute of Diabetes, Nutrition and Metabolic Diseases (Bucharest, Romania) and approved by the Ethics Committee of 'Prof. N.C. Paulescu' National Institute of Diabetes, Nutrition and Metabolic Diseases (Bucharest, Romania), and an informed consent was obtained from all the patients included in this study.

\section{Patient consent for publication}

Not applicable.

\section{Competing interests}

The authors declare that they have no competing interests.

\section{References}

1. Cox NH, Eedy DJ and Morton CA; British Association of Dermatologists: Guidelines for management of Bowen's disease. Br J Dermatol 141: 633-641, 1999.

2. Cox NH, Eedy DJ and Morton CA; Therapy Guidelines and Audit Subcommittee, British Association of Dermatologists: Guidelines for management of Bowen's disease: 2006 update. $\mathrm{Br}$ J Dermatol 156: 11-21, 2007.

3. Weinberg AS, Ogle CA and Shim EK: Metastatic cutaneous squamous cell carcinoma: An update. Dermatol Surg 33: 885-899, 2007

4. Krishnan R, Lewis A, Orengo IF and Rosen T: Pigmented Bowen's disease (squamous cell carcinoma in situ): A mimic of malignant melanoma. Dermatol Surg 27: 673-674, 2001.
5. Cameron A, Rosendahl C, Tschandl P, Riedl E and Kittler H: Dermatoscopy of pigmented Bowen's disease. J Am Acad Dermatol 62: 597-604, 2010.

6. Micali G and Lacarrubba F: Possible applications of videodermatoscopy beyond pigmented lesions. Int J Dermatol 42: 430-433, 2003.

7. Zalaudek I, Argenziano G, Di Stefani A, Ferrara G, Marghoob AA, Hofmann-Wellenhof R, Soyer HP, Braun R and Kerl H: Dermoscopy in general dermatology. Dermatology 212: 7-18, 2006.

8. Ghita MA, Caruntu C, Rosca AE, Kaleshi H, Caruntu A, Moraru L, Docea AO, Zurac S, Boda D, Neagu M, et al: Reflectance confocal microscopy and dermoscopy for in vivo, non-invasive skin imaging of superficial basal cell carcinoma. Oncol Lett 11: 3019-3024, 2016.

9. Căruntu C, Boda D, Guţu DE and Căruntu A: In vivo reflectance confocal microscopy of basal cell carcinoma with cystic degeneration. Rom J Morphol Embryol 55: 1437-1441, 2014.

10. Lupu M, Caruntu A, Caruntu C, Boda D, Moraru L, Voiculescu V and Bastian A: Non-invasive imaging of actinic cheilitis and squamous cell carcinoma of the lip. Mol Clin Oncol 8: 640-646, 2018.

11. Batani A, Brănişteanu DE, Ilie MA, Boda D, Ianosi S, Ianosi G and Caruntu C: Assessment of dermal papillary and microvascular parameters in psoriasis vulgaris using in vivo reflectance confocal microscopy. Exp Ther Med 15: 1241-1246, 2018.

12. Căruntu C, Boda D, Căruntu A, Rotaru M, Baderca F and Zurac S: In vivo imaging techniques for psoriatic lesions. Rom J Morphol Embryol 55 (Suppl): 1191-1196, 2014.

13. Swindells K, Burnett N, Rius-Diaz F, González E, Mihm MC and González S: Reflectance confocal microscopy may differentiate acute allergic and irritant contact dermatitis in vivo. J Am Acad Dermatol 50: 220-228, 2004.

14. Ardigò M, Maliszewski I, Cota C, Scope A, Sacerdoti G, Gonzalez S and Berardesca E: Preliminary evaluation of in vivo reflectance confocal microscopy features of Discoid lupus erythematosus. Br J Dermatol 156: 1196-1203, 2007.

15. Markus R, Huzaira M, Anderson RR and González S: A better potassium hydroxide preparation? In vivo diagnosis of tinea with confocal microscopy. Arch Dermatol 137: 1076-1078, 2001.

16. González S, Rajadhyaksha M, González-Serva A, White WM and Anderson RR: Confocal reflectance imaging of folliculitis in vivo: Correlation with routine histology. J Cutan Pathol 26: 201-205, 1999.

17. Diaconeasa A, Boda D, Neagu M, Constantin C, Căruntu C, Vlădău L and Guţu D: The role of confocal microscopy in the dermato-oncology practice. J Med Life 4: 63-74, 2011.

18. Ulrich M, Kanitakis J, González S, Lange-Asschenfeldt S, Stockfleth E and Roewert-Huber J: Evaluation of Bowen disease by in vivo reflectance confocal microscopy. Br J Dermatol 166: 451-453, 2012.

19. Braga JC, Paschoal FM, Blumetti TC, Bussade M, Duprat J, Landman G and Rezze GG: Hypomelanotic melanoma mimicking pigmented Bowen disease. J Am Acad Dermatol 74: e11-e13, 2016.

20. Argenziano G, Soyer HP, Chimenti S, Talamini R, Corona R, Sera F, Binder M, Cerroni L, De Rosa G, Ferrara G, et al: Dermoscopy of pigmented skin lesions: Results of a consensus meeting via the Internet. J Am Acad Dermatol 48: 679-693, 2003.

21. Argenziano G and Soyer HP: Dermoscopy of pigmented skin lesions - a valuable tool for early diagnosis of melanoma. Lancet Oncol 2: 443-449, 2001.

22. Argenziano G, Fabbrocini G, Carli P,De Giorgi V and Delfino M: Epiluminescence microscopy: Criteria of cutaneous melanoma progression. J Am Acad Dermatol 37: 68-74, 1997.

23. Ishihara Y, Saida T, Miyazaki A, Koga H, Taniguchi A, Tsuchida T, Toyama M and Ohara K: Early acral melanoma in situ: Correlation between the parallel ridge pattern on dermoscopy and microscopic features. Am J Dermatopathol 28: 21-27, 2006.

24. Argenziano G, Zalaudek I, Corona R, Sera F, Cicale L, Petrillo G, Ruocco E, Hofmann-Wellenhof R and Soyer HP: Vascular structures in skin tumors: A dermoscopy study. Arch Dermatol 140: 1485-1489, 2004.

25. Pan Y, Chamberlain AJ, Bailey M, Chong AH, Haskett M and Kelly JW: Dermatoscopy aids in the diagnosis of the solitary red scaly patch or plaque-features distinguishing superficial basal cell carcinoma, intraepidermal carcinoma, and psoriasis. J Am Acad Dermatol 59: 268-274, 2008. 
26. Kreusch $\mathrm{J}$ and Koch F: Incident light microscopic characterization of vascular patterns in skin tumors. Hautartz 47: 264-272, 1996 (In German).

27. Solovastru-Gheuca L, Vata D, Statescu L, Constantin MM and Andrese E: Skin cancer between myth and reality, yet ethically constrained. Rev Rom Bioet 12: 47-52, 2014.

28. Ragi G, Turner MS, Klein LE and Stoll HL Jr: Pigmented Bowen's disease and review of 420 Bowen's disease lesions. J Dermatol Surg Oncol 14: 765-769, 1988.

29. Firooz A, Farsi N, Rashighi-Firoozabadi M and Gorouhi F: Pigmented Bowen's disease of the finger mimicking malignant melanoma. Arch Iran Med 10: 255-257, 2007.

30. Bugatti L, Filosa G and De Angelis R: Dermoscopic observation of Bowen's disease. J Eur Acad Dermatol Venereol 18: 572-574, 2004.

31. Kittler H: Dermatoscopy: Introduction of a new algorithmic method based on pattern analysis for diagnosis of pigmented skin lesions. Dermatopathology. Pract Concept 13: 3, 2007.

32. Ulrich M, Lange-Asschenfeldt $S$ and González S: In vivo reflectance confocal microscopy for early diagnosis of nonmelanoma skin cancer. Actas Dermosifiliogr 103: 784-789, 2012.

33. Malvehy JH-MN, Costa J, Salerni G, Carrera C and Puig S: Semiology and pattern analysis in nonmelanocytic lesions. In: Reflectance Confocal Microscopy for Skin Diseases. Springer, Berlin, pp239-252, 2012.

34. Incel P, Gurel MS and Erdemir AV: Vascular patterns of nonpigmented tumoral skin lesions: Confocal perspectives. Skin Res Technol 21: 333-339, 2015.
35. Ulrich M, Maltusch A, Rius-Diaz F, Röwert-Huber J, González S, Sterry W, Stockfleth E and Astner S: Clinical applicability of in vivo reflectance confocal microscopy for the diagnosis of actinic keratoses. Dermatol Surg 34: 610-619, 2008.

36. Röwert-Huber J, Patel MJ, Forschner T, Ulrich C, Eberle J, Kerl H, Sterry W, Stockfleth E and Stockfletch E: Actinic keratosis is an early in situ squamous cell carcinoma: A proposal for reclassification. Br J Dermatol 156 (Suppl 3): 8-12, 2007.

37. Ahlgrimm-Siess V, Cao T, Oliviero M, Hofmann-Wellenhof R, Rabinovitz HS and Scope A: The vasculature of nonmelanocytic skin tumors on reflectance confocal microscopy: Vascular features of squamous cell carcinoma in situ. Arch Dermatol 147: 264, 2011.

38. Debarbieux S, Perrot JL, Cinotti E, Labeille B, Fontaine J, Douchet C, Balme B and Thomas L: Reflectance confocal microscopy of Pigmented Bowen's disease: Misleading dendritic cells. Skin Res Technol 23: 126-128, 2017.

39. Nguyen KP, Peppelman M, Hoogedoorn L, Van Erp PE and Gerritsen MP: The current role of in vivo reflectance confocal microscopy within the continuum of actinic keratosis and squamous cell carcinoma: A systematic review. Eur J Dermatol 26: 549-565, 2016.

(i) (9) This work is licensed under a Creative Common International (CC BY-NC-ND 4.0) License. 